\title{
Hypothetical metaphysics of nature
}

\author{
Michael Esfeld \\ University of Lausanne, Department of Philosophy \\ CH-1015 Lausanne, Switzerland \\ Michael-Andreas.Esfeld@unil.ch
}

(for Michael Heidelberger \& Gregor Schiemann (eds.):

The significance of the hypothetical in the natural sciences, Berlin: de Gruyter 2009)

\begin{abstract}
The paper first sketches out a reply to the underdetermination challenge and the incommensurability challenge that rebuts the sceptical conclusions of these challenges and that is sufficient to lay the ground for the project of a metaphysics of nature. That metaphysics is as hypothetical as are our scientific theories. The paper then explains how can one can argue for certain views in the metaphysics of nature based on our current fundamental physical theories, namely the commitments to a tenseless theory of time and existence instead of a tensed one, to events instead of substances, and to relations instead of intrinsic properties. Finally, the paper mentions the themes of causation, laws and dispositions.
\end{abstract}

\section{Introduction}

Whereas the philosophy of science concentrated for a long time on epistemological issues concerning the justification of knowledge claims contained in scientific theories, the project of a metaphysics of nature (Naturphilosophie in German) or metaphysics of science has gathered new momentum in recent years. The new metaphysics of nature distinguishes itself from the older essays in speculative metaphysics by being close to science: metaphysical claims are based on scientific theories. Consequently, the metaphysical claims about nature are as hypothetical as our scientific theories: there is no more certainty to be gained in metaphysics than there is in science. In other words, scientific knowledge claims are fallible and metaphysics, insofar as it draws on those claims, is as fallible as science. The idea of a metaphysics of nature close to science is, however, not without forerunners among $19^{\text {th }}$ and $20^{\text {th }}$ century philosophers. Gustav Theodor Fechner, Hermann Lotze, Eduard von Hartmann, Erich Becher, Aloys Wenzl and others have tried to overcome the speculative metaphysics of German Idealism by clinging to the science of their day. The advent of modern physics has profoundly changed the outlook of this enterprise.

Pursuing the project of a metaphysics of nature evidently presupposes the view that the pretensions to knowledge contained in our scientific theories are not baseless. In other words, some sort of scientific realism is presupposed (cf. Hawley 2006). Accordingly, in this paper, I will first sketch out a reply to scepticism about scientific knowledge that is sufficient as a basis for a metaphysics of nature. I will then discuss a few examples of positions that it is reasonable to endorse when it comes to constructing a metaphysics of nature grounded on our current fundamental physical theories. The thesis that this paper seeks to illustrate is that there is a mutual dependence between science and philosophy: philosophy in the sense of metaphysics needs science to know about what there is in the real world, and science needs 
philosophy in the sense of epistemology when it comes to developing criteria for the justification of science's pretensions to knowledge in general and the assessment of knowledge claims contained in specific scientific theories.

Following David Papineau, one can divide the philosophy of science into two broad areas:

The epistemology of science deals with the justification of claims to scientific knowledge. The metaphysics of science investigates philosophically puzzling features of the world described by science. In effect, the epistemology of science asks whether scientific theories are true, whereas the metaphysics of science considers what it would tell us about the world if they were.

(Papineau 1996, 1)

In the next two sections, I shall outline a reply to the two main challenges to scientific realism stemming from underdetermination and from incommensurability. The reply to be developed here is sufficient to justify engaging in the project of a metaphysics of nature. The second half of the paper goes into that project, sketching out three metaphysical claims grounded on our current fundamental physical theories, namely a tenseless view of time and existence (section 4), a metaphysics of events (section 5) and a metaphysics of relations (section 6). Finally, I shall briefly touch upon the topics of causation, laws and dispositions (section 7).

\section{The underdetermination challenge}

If one pursues the project of a metaphysics of nature based on science, one presupposes that science is in principle capable of providing a cognitive access to the constitution of nature. Since there are different scientific theories - or different models or interpretations of a given scientific theory - that are incompatible with each other, that presupposition amounts to the thesis that we have methods at our disposition which enable a rational evaluation of the claims about the constitution of nature contained in different and incompatible scientific theories (or incompatible models or interpretations of a given theory).

That presupposition faces a challenge from confirmation holism and its consequence, namely that scientific theories are underdetermined by experience. Confirmation holism is the thesis that experience does not confirm or refute single propositions taken in isolation, but always a whole network of propositions or a whole theory. Confirmation holism goes back to Duhem (1914) - (English translation Duhem 1954; see part 2, chapter 6) - and Quine (1953). It is known as the Duhem-Quine thesis. The main argument is that whenever a single proposition is taken to be refuted by experience, there are background propositions available. There is always the logical possibility to change the truth-values attributed to some of these background propositions such that one can continue to hold on to the original proposition despite the recalcitrant experience. Confirmation holism therefore implies that theory is underdetermined by experience: for any given body of experience, it is logically possible to construct an indefinite number of theories that are incompatible with each other, but that are all in accordance with the experience - that is, it is possible to infer from each of these theories the observational propositions describing the experience in question.

Logical possibility, however, does not mean that there always are at least two rival theories that are equally credible. It is trivial that one can always construct a rival theory that is in accordance with the experimental data by ad hoc modifications of a given theory. This has no implications for the issue of which theory it is rational to accept. One may construe the claims Quine makes in "Two dogmas" as meaning, among other things, that for any proposition, circumstances are conceivable in which it is rational to abandon the proposition 
in question in order to adapt a given system of propositions to new experimental data. The issue of whether or not it is rational to renounce certain propositions regarded as logical laws - such as the law of the excluded middle - to accommodate experience in the domain of quantum physics is a case in point (see Quine 1953, 43). However, this does not imply that for any given body of experience we can conceive at least two rival theories that are both in accordance with the experience, without there being any further rational criteria that distinguish one of these theories as a better candidate for a true theory than the other one(s) (see Laudan 1996, chapter 2, against any such claim based on underdetermination).

Whereas the history of science provides many examples of successor theories being incompatible with their predecessors, it is rare to find an example of two or more contemporary theories that contradict themselves in their ontological commitments, that make exactly the same experimental predictions and none of which is an ad hoc modification of a given theory. Quantum physics is one such rare example. Standard quantum mechanics as set out, for instance, in von Neumann (1932) - (English translation von Neumann 1955) - and Bohm's rival theory - (first version Bohm 1952, last major publication by Bohm himself Bohm \& Hiley 1993) - make exactly the same empirical predictions. Nonetheless, there are at least two criteria that one can invoke in favour of a rational evaluation of any two such theories. First, if they agree in their experimental predictions at a given time, this does not imply that it is impossible to conceive of an area in which the two theories may distinguish themselves by different predictions of experimental results (see Laudan \& Leplin 1991, 451455, reprinted in Laudan 1996, 56-59). For instance, in the case of standard quantum mechanics vs. Bohm's theory, the domain of quantum fields may constitute such an area: it is an open question whether there can be a Bohmian theory that is equivalent to quantum field theory (but see Bohm, Hiley \& Kaloyerou 1987 and Huggett \& Weingard 1994, 382-387).

Moreover, if there are two rival theories that agree in their predictions of experimental results, they are rival theories because their ontological commitments are different. It is always possible to evaluate these commitments. Confirmation holism speaks against foundationalism in epistemology: experience is not a foundation of knowledge in the sense of being able on its own to justify scientific theories, for the same experience can be accounted for by rival theories. The logical possibility of such rival theories is sufficient to refute such a foundationalism. Confirmation holism therefore is linked to justification holism, that is, the view that a proposition is justified if and only if it is coherent with other propositions in a whole network of propositions (a whole theory). We can always employ the coherence criterion of justification holism to assess the ontological commitments of rival theories that agree in their experimental predictions. The coherence criterion examines two or more such rival theories against the background of our other knowledge, seeking to establish which one of these rival theories fits better into a coherent system of our knowledge as a whole. The idea behind that criterion is that explanation is linked to coherence: each of the rival theories seeks to explain a certain range of phenomena. These explanations, in turn, can be evaluated as to which one is more likely to contain a true description of entities and their connections in the world, given what else we know about the world.

In this vein, the adherent to standard quantum mechanics can point out against the Bohmian that the quantum potential that Bohm's theory has to stipulate in order to reproduce the experimental predictions of standard quantum mechanics is a very implausible assumption against the background of our other physical knowledge: it is entirely different from a 
potential in classical physics, and the only argument available for its introduction is the intention to reproduce the experimental predictions of standard quantum mechanics. To counter that objection, the Bohmian, in turn, can point to the measurement problem into which standard quantum mechanics runs. (The question, however, is whether ontological assumptions such as those ones contained in Bohm's theory are necessary in order to solve that problem).

But what about two or more rival accounts, each of which is conceived as a complete theory of the world? One may think of an interpretation of quantum theory in the style of Everett (1957) according to which everything is a quantum object, subject to quantum entanglement, and no quantum state reductions occur, not even in measurement. There exists only one entangled quantum state of the world at a time that includes all possible values that all properties can take as really existing in infinitely many different branches of the world. On the other hand, there is an interpretation of quantum theory that includes state reductions and thus a dissolution of entanglement (the most precise proposal in that respect goes back to Ghirardi, Rimini \& Weber 1986). Consequently, there really are classical objects and classical properties that have precise numerical values in the world. Again, it is possible to evaluate and to compare the ontological commitments of any such rival complete worldviews. For instance, the worldview of universal quantum entanglement commits us in the last resort to the view that each person has infinitely many minds, existing in different branches of the universe (see Albert \& Loewer 1988 and Lockwood 1989, chapters 12 \& 13). In any case, it implies that each single physical object exists infinitely many times in different branches of the universe, having different values of its time-dependent properties in these branches. One may voice with good reason reservations about such extravagant commitments.

Such an assessment of ontological commitments does not have to lead to an uncontroversial result that is accepted by all parties. There rarely are such results in philosophical debates. The interpretation of quantum mechanics again is a case in point. But this does not hinder there being sound arguments which suggest that certain theories - or models or interpretations of a given theory - are the best hypotheses about the constitution of nature or a certain domain of nature that we can put forward given the state of our scientific knowledge. Hence, the underdetermination challenge does not undermine the claim that science is in principle capable of providing a cognitive access to the constitution of nature.

\section{The incommensurability challenge}

Apart from confirmation holism and justification holism, there is a further form of holism that leads to another challenge to the knowledge claims contained in scientific theories: semantic holism. The basic idea is that the content (meaning) of a concept is given by its position in a whole network of concepts, that is, a whole theory. In short, the content of a concept consists in its inferential relations to other concepts (cf. Esfeld 2001, chapter 2). Semantic holism is widely accepted at least with respect to theoretical concepts. For instance, the concept "electron" cannot be introduced by pointing to electrons. It is introduced by indicating its inferential relations to other concepts in a whole theory. The theory as a whole then has certain observational consequences.

Semantic holism implies that there is no separation between issues concerning factual matters and issues concerning conceptual content (meaning). Changes in our views as to what the world is like go together with changes in conceptual content. The reason is that a theory 
change entails a change in some inferential relations - that is, according to semantic holism, a change in conceptual content. For instance, in Newton's theory, gravitation is conceived of as a force that acts at a distance. In other words, there is an inferential link between the concept of gravitation and the concept of action at a distance. In general relativity, by contrast, gravitation is conceived of as an effect of the curvature of space-time. Hence, the inferential link from gravitation to action as a distance is cut off and replaced with a link from gravitation to space-time curvature and effects that the latter has. Another prominent example is the change in the content (inferential relations) of the concept "electron" from prescientific theories via classical field theory to quantum mechanics and quantum field theory. Examples such as these ones are taken to show that there can be a radical conceptual change when one theory is replaced with another.

Such examples drive the claim of semantic incommensurability put forward by Kuhn (1962, chapters $9 \&$ 10) and Feyerabend (1962), which is based on semantic holism. For instance, the concept of gravitation as action at a distance is incommensurable with the concept of gravitation as an effect of space-time curvature, because these two concepts do not have any significant scientific inferences in common. There is a radical conceptual change from Newton's theory of gravitation to Einstein's theory of gravitation: the language of general relativity does not even have the conceptual means at its disposal to express the idea of an action at a distance. In this sense, Newton's concept of gravitation cannot be translated into general relativity.

The thesis of semantic incommensurability calls into question whether there is progress on the level of our views about the constitution of nature: if the proper concepts of a new theory are incommensurable with those of its predecessor, then there is no basis for claiming that the new theory makes cognitive progress in revealing the constitution of nature. Kuhn goes as far as suggesting the replacement of the idea of science making cognitive progress with the idea of changes of scientific theories not being directed to any goal (1962, chapter 13). Incommensurability is thus grist to the mills of what is known as the argument from pessimistic meta-induction (Laudan 1981 and 1984, in particular 157), although that argument is not committed to the incommensurability thesis: our past theories have turned out to be false. There is no reason to suppose that our current theories will endure a better fate. We are thus not justified in supposing that there is cognitive progress in the history of science.

There is an easy Popperian answer to the argument from pessimistic meta-induction: most of our past theories have indeed been falsified. But falsification is the means to make cognitive progress. By replacing our old theories with new ones we correct the errors that the old theories contain. We make cognitive progress in the sense that we come closer and closer to the truth about the constitution of nature by falsifying our old theories and replacing them with new ones (see Popper 1959, in particular chapters 4 \& 10). This Popperian reply presupposes, however, that the newer theories do not only improve on the older theories as regards the range and the accuracy of predictions, but also that the theoretical views which the newer theories offer are comparable with the ones of the older theories. The views about the constitution of nature contained in the newer theories are surely incompatible with the ones of the older theories: they contradict them. Nonetheless, it is possible to compare the proper concepts of the newer theories with the proper concepts of the older ones. Being in a position to carry out such a comparison is a necessary condition for being able to argue that we come closer and closer to the truth through falsification. Otherwise, there would only be theory 
replacement, but no continuous progress towards truth. It is here that the argument from semantic incommensurability comes in: that argument seeks to establish that this presupposition of comparability is not satisfied. In that sense, the thesis of semantic incommensurability is much more radical than the conclusion of the argument from pessimistic meta-induction.

However, in order to counter that thesis, one can point out that there are precise logical relations between the formalisms - that is, the mathematical equations - of any major mature physical theories that succeed each other in the history of science. It is well known that the formalisms of the main mature classical physical theories before the $20^{\text {th }}$ century can be reconstructed from the formalisms of their successor theories in relativity physics and quantum physics. More precisely, it is possible to derive within the formalisms of the newer theories an image of the formalisms of the older theories that reproduces the predictions of the older theories in the cases where one can neglect certain quantities: if one carries out a mathematical operation that lets the quantum of action approach zero $(\mathrm{h} \rightarrow 0)$ or a mathematical operation that lets the velocity of light approach infinity (so that $1 / \mathrm{c} \rightarrow 0$ ) one can reproduce within quantum mechanics or special relativity the predictions of classical mechanics. The situations in which classical mechanics is applied successfully are such that one can for all practical purposes proceed as if the quantum of action were zero or as if the velocity of light were infinite; the objects dealt with are very big in comparison to the quantum of action, and the velocities are very small in comparison to the velocity of light. In fact, the matter is more complicated and requires a detailed mathematical examination. These examples are only meant to give a rough idea of how one seeks to reconstruct the formalism of classical mechanics from the formalisms of its successor theories. This possibility to reconstruct the formalism of classical mechanics explains why the predictions of classical mechanics are successful in certain areas.

Some philosophers take the possibility of such a reconstruction of the formalism of the older theory from the formalism of the newer theory to be a sufficient basis for claiming that the concepts of the older theory can be integrated into the concepts of the newer theory. They go as far as maintaining that the older theories are reduced to the newer ones (see, in particular, Schaffner 1967, Hooker 1981, part I, § 3, and 2004, sections 1-3). However, that conclusion cannot be warranted on this basis alone. The formal structure of the newer theories is very different from the one of the older theories. Special relativity, for instance, is fundamentally distinct from its predecessor theories in posing the principle that the velocity of light is finite and constant, independently of any reference frame. The same goes for quantum mechanics in comparison to classical mechanics; that is why quantum mechanics, in distinction to classical mechanics, raises a number of notorious problems of interpretation. The fact that the formal structure is considerably different means that there is a great difference on the level of the views of the constitution of nature that both theories offer. What is therefore needed is not only a reconstruction of the formalism, but also a reconstruction of the concepts of the predecessor theories from the concepts of the successor theories.

There is no general rule regarding how to carry out such a reconstruction of concepts. To my mind, the examples of major theory replacements from classical to contemporary physics allow us to distinguish three cases - a smooth case of reduction (1), an intermediate case of reconstruction without reduction (2) and the radical case of incommensurability (3). 
(1) Consider the example of classical mechanics and quantum mechanics. Assume a version of quantum mechanics that includes a dynamics which leads to definite numerical values as measurement outcomes (the most elaborate proposal in that respect goes back to Ghirardi, Rimini \& Weber 1986, although there still are a number of physical problems with that proposal). In that case, quantum theory includes the description of a transition from quantum states, characterized by superpositions and entanglement, to classical states. There is, of course, a considerable conceptual change from classical mechanics to quantum mechanics. But this change does not amount to semantic incommensurability. The change concerns the view of what is fundamental in nature. Quantum mechanics shows that the assumptions of classical mechanics about what is fundamental in nature are false; nonetheless, classical concepts can be derived within quantum mechanics, having a limited domain of applicability: they apply as soon as quantum superpositions and entanglements are dissolved, and quantum mechanics, on the version proposed by Ghirardi, Rimini \& Weber (1986), includes a dynamics for this dissolution (state reductions). Generally speaking, this is a case where the concepts of the older theory can be integrated into the concepts of the newer theory, having a limited domain of applicability within the domain that the newer theory covers.

(2) The case is different if one maintains a version of quantum mechanics that does not acknowledge definite numerical values as measurement outcomes so that there are no state reductions. Instead, everything that there is in the physical world is subject to quantum entanglement, and there is only a universal quantum state of the world developing in time (that version, which is based exclusively on the Schrödinger dynamics, goes back to Everett 1957). Definite numerical values of physical quantities as measurement outcomes are merely the way in which the world appears to local observers whose cognitive access is confined to a particular branch of the universe. Definite numerical values as measurement outcomes - and classical properties in general - are relative to a certain state of consciousness of a local observer. Apart from that particular branch of the universe, there are infinitely many other branches of the universe in which other numerical values of the physical properties in question exist relative to other states of mind of the observer. The universal quantum state of the world includes all these branches.

In that case, there is no domain of the world to which the concepts of classical physics apply, for there is nothing that is not subject to quantum entanglement. What classical physics says about the world is false and beside the point. Nonetheless, one can reconstruct the concepts of classical physics on the basis of the concepts of quantum theory. Given the universal quantum state of the world that includes infinitely many different branches of the world in which the same physical objects exist in different relative states, one can reconstruct the concepts of classical physics as describing how the world appears to local observers. The mistake of classical physics is to take that appearance for the physical reality. Generally speaking, given the wider conceptual framework of the successor theory, one can reconstruct the concepts of the predecessor theory within that framework, although these concepts do not yield any true or approximately true descriptions of what there is in the world. Instead, they describe how the world appears to a local observer with limited cognitive access.

The case of classical mechanics and special relativity is in a certain manner conceptually similar. If the velocity of light were infinite, the distinction between events that are separated from each other by a spacelike interval, by a timelike interval and by a lightlike interval 
would collapse. Spatial and temporal distances, including notably the relation of similarity between events, would no longer depend on a frame of reference. However, given that the velocity of light is finite and constant, there is no domain to which the spatio-temporal concepts of classical mechanics apply. Nonetheless, one can reconstruct these concepts within the framework that is given by the principle of the velocity of light being finite and constant, showing how these concepts describe how the world appears to an observer within a given frame of reference. The observer takes these concepts to be objective, not realizing that they depend on a frame of reference (that is privileged for her, but not privileged in a general manner).

(3) Consider the case of Newton's theory of gravitation and general relativity. Although the predictions nearly coincide within a certain domain, there is no possibility to reconstruct within general relativity the concept of an action at a distance. It is not possible to reconstruct that concept as describing how the world appears to a local observer. That concept points out a consequence to which Newton's theory of gravitation is committed; it does not belong to the repertoire of a local observer. In short, the view of there being an action at a distance is simply abandoned and replaced with a theory of curved space-time according to which gravitation is an effect of space-time curvature.

In none of the three types of cases considered, do the concepts of the predecessor theory come close to or approximate the concepts of the successor theory. Furthermore, all these cases are much more complicated than is suggested by the mathematical operation of letting a particular quantity approach zero in order to derive an image of the formalism of the old theory within the formalism of the new theory. The reason is the difference in the formal structure of the successor theories in comparison to their predecessors. Even in the case of quantum mechanics and classical mechanics, there is no question of a simple transformation of the reconstruction of the formalism into a reconstruction, or even a reduction, of the concepts. Everything depends on the way in which one interprets quantum mechanics.

Nonetheless, only the third type of case is a case of semantic incommensurability. The thesis that semantic incommensurability is widespread is unfounded. The first type of case is a case of theory reduction: if quantum mechanics includes a dynamics for a transition from superposed and entangled quantum states to classical states, then it is possible to reduce classical mechanics to that version of quantum mechanics; for it is then possible to derive within quantum mechanics concepts that match the role (inferential relations) of the proper concepts of classical mechanics within a certain domain. The second type of case is situated somewhere in between: there is no domain in which the concepts of the predecessor theory truly apply or are approximately true, but it is possible to reconstruct the concepts of the old theory within the framework of the new theory, namely as describing how the world appears to observers with a limited cognitive access.

Already the second type of case is sufficient to ground the claim that there is not a simple theory replacement in the history of science, but that the successor theories come closer to the truth than their predecessor ones (although we do not have at our disposal a universally accepted, precise definition of the notion of coming close to the truth). One can maintain that there is an approximation to a true and complete theory of the domain in question - or even nature as a whole - in the following sense: the common cases of replacing the old theories with new ones amount to an enlargement of our point of view, because it is possible to reconstruct within the new theory the proper concepts of the old theory as describing the way 
in which the world appears to a local observer with a limited cognitive access. In replacing our old theories with better ones, we decrease the cognitive limits of our point of view, coming closer to an objective description, that is, a description and explanation of the world from a point of view of nowhere. Science is the ambitious project of transgressing our cognitive limits, given that we are finite thinking beings confined to a very small spatiotemporal region of the universe, seeking to reach an objective description of the world.

To sum up, the incommensurability challenge and the underdetermination challenge can be countered in such a way that they do not undermine the project of a metaphysics of nature based on science. The second type of case considered in this section is already sufficient as a basis for that project. In the remaining sections of the paper, I shall consider four claims within the scope of that project and their link with our current scientific theories.

\section{The tenseless theory of time and existence}

There are two rival theories of time as well as of existence. According to the tensed theory of existence, existence is relative to time in the sense that only that what is present - or only that what is present and that what is past - exists. What is in the future does not exist as yet, and, according to some versions of this theory, what is past does not exist any more. The tensed theory of existence implies the tensed theory of time according to which the past, the present and the future are objective modes of time, being out there in the world. The tensed theory of time, however, does not imply the tensed theory of existence.

Opposed to the tensed theory of time is the tenseless theory of time which claims that there are only temporal relations of being earlier than, simultaneous with and later than among events, but no objective modes of past, present and future. The tenseless theory of time implies the tenseless theory of existence according to which existence is not relative to a location in time in the same way as it is not relative to a location in space: everything that there is in space and time simply exists. The tenseless theory of existence, however, does not imply the tenseless theory of time.

There are philosophical arguments in favour of both these theories of time and existence. The case can be settled by taking science into account. The relevant scientific theory is special relativity. Special relativity shows that there is no simultaneity between events independently of a reference frame. Any event - consisting in physical properties that occur at a space-time point - that is supposed to be simultaneous with other events is so only relative to a reference frame, and there is no globally preferred reference frame. Thus, there is no objective "now"in the same way as there is no objective "here". For any space-time point, it can be claimed that it is "present" in the same way as it can be claimed that it is "here" (see, for instance, Dorato 1995, chapters 11 to 13, in particular pp. 186-187, 210). The reason is that, according to special relativity, spatial as well as temporal distances between events are relative to a reference frame. Invariant with respect to the choice of a reference frame is only the fourdimensional, spatio-temporal distance between any two events (or points of space-time). That is the reason why special relativity is taken to show that space and time are united in a fourdimensional entity, space-time.

Special relativity hence makes a case for the tenseless theories of time and existence. Since spatial and temporal distances are relative to a reference frame, there is no basis in the physical world for upholding a tensed theory of time or existence (see Saunders 2002). Special relativity does not exclude that there is a direction of time in the universe accounting 
for irreversible processes (see Maudlin 2007, 108-110, 115-117); but it contains a decisive argument against the assumption that there are objective modes of time. Nonetheless, the tenseless theories of time and existence belong to the metaphysics of nature. It is logically possible to rescue the idea of an objective present by introducing the notion of one globally privileged reference frame. That notion does not contradict special relativity. The point is that it is entirely ad hoc.

Furthermore, basing a tenseless theory of time and existence on special relativity is a hypothetical claim, since it depends on one particular scientific theory that may be superseded in the future. General relativity - and notably its application in cosmology - goes beyond special relativity; but it does not change anything with respect to what special relativity says about the relativity of spatial and temporal distances given the principle of general covariance that excludes that there is a globally privileged coordinate system. However, it cannot be ruled out that a future theory that transcends general relativity may change that matter.

\section{Events instead of substances}

The tenseless theories of time and existence, based on the physics of special relativity, result in what is known as the view of the world as a block universe: the whole of four-dimensional space-time is a single block so to speak, including time; everything that there is exists at a space-time point or region. What is the content of the block universe?

In metaphysics, it is common to draw a distinction between substances and events. Substances persist as a whole for a certain time. They have spatial parts (unless they are atoms in a literal sense), but they do not have any temporal parts. Relying on physics, an event can be conceived as consisting in the physical properties that occur at a space-time point. Let us leave open whether these physical properties literally are properties of spacetime points or whether they are properties of matter (e.g. fields) located at space-time points. Continuous sequences of events are processes. Processes have spatial as well as temporal parts. Four-dimensional objects such as processes are commonly conceived of as perdurants, persisting by having spatial as well as temporal parts, whereas three-dimensional objects such as substances are conceived as endurants, persisting as a whole for a certain time, having no temporal parts.

Common sense admits both substances and processes. A volcano, for instance, is regarded as a substance, persisting for a certain time by having no temporal parts, but only spatial parts. The eruption of a volcano, by contrast, is a process, persisting for a time by having temporal as well as spatial parts. The eruption can, for instance, be first mild and then heavy.

Events and processes cannot be dispensed with in metaphysics. Even if it were possible to conceive all events as consisting in changes of the properties of substances, there would be a dualism of substances and events qua changes in the properties of substances. However, it may be possible to do without substances, recognizing only events and processes. (There is an ambiguity in the notion of a substance: if one regards space-time points as substances, they are not substances in the sense of endurants, but four-dimensional objects that have neither spatial nor temporal parts; they are not in space and time, but they are what makes up spacetime). Again, there is a philosophical dispute as to whether or not one should admit substances in addition to events. Again, science is relevant to that dispute.

If one switches from a physics of three-dimensional space to a physics of four-dimensional space-time (block universe), there no longer is any need to admit substances as the objects 
that are the enduring foundation of change, change being the change of properties of substances, motion being change in the location of substances. Moreover, there is no need to conceive of the transtemporal identity of objects as the identity of substances in time, because substances do not have temporal parts.

In the metaphysics of the block universe, transtemporal identity can be accounted for in terms of genidentity, that is, sequences of events that have the same or similar properties. In other words, the transtemporal identity of any physical object is explained by the fact that the object is a process whose temporal parts form a continuous sequence, exhibiting similar physical properties. As regards motion, what common sense considers as the motion of a substance through three-dimensional space is explained as a continuous sequence of spacetime points or regions that possess a similar physical content (a world line). Change is different physical properties instantiated at points or regions of space-time forming such a continuous sequence.

Hence, against the background of special relativity, it is in the first place the philosophical arguments of coherence and parsimony that speak in favour of a metaphysics of events - and processes (perdurants) -, admitting no substances (endurants): if one makes the step to a metaphysics of a four-dimensional block universe, it is simply not coherent to recognize three-dimensional substances among the content of the block universe. Four-dimensional events and their sequences (processes) have to be accepted anyway, and they are sufficient as the furniture of the universe (see Sider 2001 as regards the philosophical arguments).

Moreover, in recent years, arguments have been developed to the effect that admitting three-dimensional substances with spatial, but no temporal parts, is not consistent with special relativity. According to special relativity, the spatial distances between points depend on a reference frame. Consequently, if one subscribes to an ontology according to which there are three-dimensional macroscopic substances, their spatial figure varies from one frame of reference to another one, because the spatial distances between the points that the substance in question occupies depend on a reference frame. If, by contrast, physical objects are fourdimensional perdurants, their figure in four-dimensional space-time is not relative to a reference frame (see Balashov 1999 as well as Hales \& Johnson 2003). A further argument makes the following point: since simultaneity is relative to a reference frame, a metaphysics of enduring three-dimensional objects cannot come up with a convincing theory of the coexistence (copresence) of objects. By contrast, a metaphysics of perduring fourdimensional objects, which have temporal parts, can easily include a theory of coexistence: any two four-dimensional objects coexist if and only if they have parts that are separated by a space-like interval (see Balashov 2000 and the discussion between Gilmore 2002 and Balashov 2005).

These arguments put the case against three-dimensional substances on a par with the case against objective simultaneity based on special relativity. Nonetheless, in any case, a metaphysics of events based on special and general relativity is as hypothetical as the tenseless theory of time and existence based on these scientific theories. They both presuppose that there is no globally preferred reference frame, and it cannot be excluded that the future development of science may introduce notions that rehabilitate the notion of a single objective temporal order in the universe. 


\section{6. $\quad$ Relations instead of intrinsic properties}

Up to now, I have argued in favour of the view of the world consisting in the last resort in the distribution of fundamental physical properties at space-time points over the whole of spacetime, forming continuous sequences that are processes and that can be regarded as physical objects (albeit no substances in the sense of things that do not have temporal parts). Quantum physics can be seen in the first place as adding something to this view concerning the physical properties. Whereas special and general relativity can be conceived as theories about spacetime, quantum physics is concerned with matter.

It is often taken for granted that the fundamental physical properties, instantiated at spacetime points, are intrinsic properties. Intrinsic are all and only those properties that an object has irrespective of whether or not there are other contingent objects; in brief, having or lacking an intrinsic property is independent of accompaniment or loneliness (see Langton \& Lewis 1998). All other properties are extrinsic, consisting in the object bearing certain relations to other objects.

Quantum physics is usually conceived of in terms of states of physical objects. The state of an object at a time can be regarded as encapsulating the properties that the object has at that time. The most striking feature of quantum theory is that the states of several objects can be entangled. In fact, starting from the formalism of quantum theory, it is to be expected that whenever one considers a complex object that consists of two or more quantum objects, the states of these objects are entangled. Entanglement is to say that it is not the case that each of the objects has a state separately. On the contrary, only the whole, that is, the complex object composed of two or more objects, is in a precise state (called a "pure state"). Philosophers of physics therefore speak of non-separability (Howard 1989) or relational holism (Teller 1986), since entanglement consists in certain relations among quantum objects.

These relations cannot be traced back to intrinsic properties of the physical objects in question. There are no intrinsic properties of the related quantum objects on which the relations of entanglement could supervene. Quantum physics can therefore be taken to suggest a metaphysics of relations, known as structural realism: insofar as quantum physics is concerned, the fundamental physical properties consist in certain relations instead of being intrinsic properties (see French \& Ladyman 2003, Esfeld 2004 and Ladyman \& Ross 2007, chapter 3; see furthermore Lyre 2004 as regards gauge theories and Esfeld \& Lam 2008 as regards structural realism with respect to general relativity).

Again, this is a conclusion belonging to the metaphysics of nature. This conclusion could be avoided by postulating intrinsic properties in the form of hidden variables that restore separability (that is, properties of quantum objects that are there, but whose value we cannot know). However, since the discovery of the theorem of John Bell (1964), it is clear that one would have to pay a high metaphysical as well as physical price for admitting hidden variables of that kind. (The only elaborate account of quantum physics in terms of hidden variables, Bohm's theory, does not fall within the scope of that criticism, for Bohm himself interprets his theory in terms of relations and holism rather than in terms of intrinsic properties; see Bohm \& Hiley 1993).

Nonetheless, again, a metaphysics of relations based on quantum physics is hypothetical. Quantum physics as it stands is definitely not the last word on matter. There is the notorious measurement problem, and there is the problem of the unification of quantum field theory and general relativity. If the project of unifying quantum field theory and general relativity 
succeeds, the resulting scientific theory will have important repercussions for our view of nature. Nonetheless, whatever may be the future fundamental physical theory that achieves a unified treatment of the phenomena that are currently considered by two different theories, it would be unreasonable to expect that future theory to go back behind the unification of space and time as considered by general relativity or the holism that quantum entanglement manifests.

\section{Causation, laws and dispositions}

One of the main themes of the metaphysics of nature is causation. What, if any, are the physical foundations of causation? The most developed proposal for a theory of causation that seeks to be close to physics is the transference theory according to which, in brief, a causal process consists in the exchange of a conserved physical quantity such as energy (Salmon 1998, chapters 1, 12, 16 and 18, Dowe 2000). However, that theory is modelled along the lines of Newtonian mechanics and special relativity. It faces serious difficulties from general relativity (see Rueger 1998, Curiel 2000, and Lam 2005), not to mention the holism of quantum theory.

Moreover, even if a proposal for a physical account of causation could be developed with success, it would not automatically settle the main issue in the metaphysics of causation, that is, the opposition between a Humean view of causation and a power view of causation. According to the Humean view, causation is nothing but a contingent regular pattern of coinstantiation of events of the same types. The Humean could employ a physical account of causation in order to distinguish the regularity patterns that are causal from the regularity patterns that are not causal. According to the power view of causation, by contrast, there are causal powers in the world that establish a necessary connection between causes and their effects (see notably Harré \& Madden 1975, in particular chapter 5, and Shoemaker 1980). The friend of causal powers could interpret a physical account of causation as identifying the fundamental manifestations of such causal powers in the world.

The view that one takes on causation has implications for one's views about laws and dispositions: for the Humean, laws are contingent regularity patterns, whereas for the friend of causal powers, they are metaphysically necessary, deriving from the causal powers, which establish necessary connections. The Humean eliminates dispositions in favour of categorical properties, reducing statements about dispositions to counterfactual conditionals that are made true by the distribution of categorical properties in the world, in virtue of that distribution exhibiting certain regularity patterns (see e.g. Sparber 2006). For the friend of causal powers, by contrast, dispositions in the form of powers are fundamental physical properties.

Both sides in this debate claim support from physics. Since Russell's famous paper denouncing the notion of causation as production (Russell 1912), some Humeans maintain that the physical theories of the $20^{\text {th }}$ century do not admit of any other sort of causation than Humean regularity patterns in the distribution of the fundamental physical properties (see e.g. Field 2003, section 1, and Norton 2007). However, the friends of powers retort that physics cannot specify the properties it deals with beyond causal-structural equivalence, thus suggesting the conclusion that the physical properties are themselves causal properties and hence powers (see notably Shoemaker 1980 and Mumford 2006). Moreover, talk in dispositional terms is widespread in fundamental physics, notably with respect to the objective probabilities of quantum theory (see Suárez 2007 for interpreting these probabilities 
in terms of propensities), but also applicable to general relativity, since space-time includes gravitation and thus a physical force (see e.g. Bartels 1996, 37-38, Bartels 2009 and Bird 2009 , section 2.3). Nonetheless, the interpretation of quantum probabilities in terms of propensities is by no means mandatory, and the fact that physics cannot specify the properties it deals with beyond causal-structural equivalence can also be accommodated within a structural realism that commits itself to categorical structures only (cf. e.g. Psillos 2006, section 6, and Sparber 2009, chapter 4).

Hence, there is a difference between themes such as the tenseless theory of time and existence vs. the tensed theory, events vs. substances, relations vs. intrinsic properties on the one hand and causation, disposition and laws on the other. As regards the former themes, our current fundamental physical theories establish certain metaphysical commitments on all reasonable standards of interpretation - namely the commitments to a tenseless theory of time and existence, to events and to relations. As regards the latter themes, there is no such direct route from physics to metaphysical conclusions. Nonetheless, physics is pertinent when it comes to arguments for the one or the other position on these issues, and it may well turn out that one can make a cogent case for one particular position on causation, laws and dispositions by drawing on physics and science in general (see Esfeld 2008, chapter 5, for an argument for the power view of causation based on physics as well as the overall coherence of our scientific knowledge and Esfeld 2009 for the relationship with physical structures).

In conclusion, there is, as stated in the introduction, a mutual dependence between science and philosophy: science needs philosophy in the sense of epistemology in order to assess the knowledge claims contained in scientific theories (as illustrated by the discussion of the underdetermination and the incommensurability challenges in sections 2 and 3). Philosophy in the sense of metaphysics needs science in order not to make claims about the world that do not stand up to scientific scrutiny. Nonetheless, science does not directly imply a particular metaphysics of nature. However, there are clear cases in which scientific theories strongly suggest on all reasonable criteria of interpretation certain metaphysical positions - such as the cases of the tenseless theory of time and existence vs. the tensed theory, events vs. substances and relations vs. intrinsic properties. Dependence on science makes the metaphysics of nature as fallible and hypothetical as scientific theories.

\section{References}

Albert, David Z. and Loewer, Barry (1988): "Interpreting the many worlds interpretation”. Synthese 77, pp. 195213.

Balashov, Yuri (1999): "Relativistic objects”. Noûs 33, pp. 644-662.

Balashov, Yuri (2000): “Enduring and perduring objects in Minkowski space-time”. Philosophical Studies 99, pp. 129-166.

Balashov, Yuri (2005): "Special relativity, coexistence and temporal parts: a reply to Gilmore”. Philosophical Studies 124, pp. 1-40.

Bartels, Andreas (1996): "Modern essentialism and the problem of individuation of spacetime points". Erkenntnis 45, pp. 25-43.

Bartels, Andreas (2009): “Dispositionen in Raumzeit-Theorien”. In: C. F. Gethmann (ed.): Lebenswelt und Wissenschaft. XXI. Deutscher Kongress für Philosophie. Kolloquien. Hamburg: Meiner.

Bell, John S. (1964): “On the Einstein-Podolsky-Rosen-paradox”. Physics 1, pp. 195-200.

Bird, Alexander (2009): "Structural properties revisited". In: T. Handfield (ed.): Dispositions and causes. Oxford: Oxford University Press. 
Bohm, David (1952): “A suggested interpretation of the quantum theory in terms of 'hidden' variables". Physical Review 85, pp. 166-193.

Bohm, David \& Hiley, Basil (1993): The undivided universe. An ontological interpretation of quantum theory. London: Routledge.

Bohm, David, Hiley, Basil \& Kaloyerou, P. N. (1987): “A causal interpretation of quantum fields”. Physics Reports 144,pp. 349-375.

Curiel, Erik (2000): “The constraints general relativity places on physicalist accounts of causality”. Theoria 15 , pp. 33-58.

Dorato, Mauro (1995): Time and reality. Spacetime physics and the objectivity of temporal becoming. Bologna: Cooperativa Libraria Universitaria Editrice Bologna.

Dowe, Phil (2000): Physical causation. Cambridge: Cambridge University Press.

Duhem, Pierre (1914): La théorie physique. Son objet - sa structure. Paris: Vrin. 2nd edition.

Duhem, Pierre (1954): The aim and structure of physical theory. Translated by Philip P. Wiener. Princeton: Princeton University Press.

Esfeld, Michael (2001): Holism in philosophy of mind and philosophy of physics. Dordrecht: Kluwer.

Esfeld, Michael (2004): "Quantum entanglement and a metaphysics of relations". Studies in History and Philosophy of Modern Physics 35B, pp. 601-617.

Esfeld, Michael (2008): Naturphilosophie als Metaphysik der Natur. Frankfurt (Main): Suhrkamp.

Esfeld, Michael (2009): "The modal nature of structures in ontic structural realism”. International Studies in the Philosophy of Science 23.

Esfeld, Michael \& Lam, Vincent (2008): “Moderate structural realism about space-time”. Synthese 160, pp. 2746.

Everett, Hugh (1957): “'Relative state' formulation of quantum mechanics”. Reviews of Modern Physics 29, pp. 454-462. Reprinted in B. S. DeWitt and N. Graham (eds.): The many-worlds interpretation of quantum mechanics. Princeton: Princeton University Press 1973. Pp. 141-149.

Feyerabend, Paul K. (1962): "Explanation, reduction, and empiricism". In: H. Feigl \& G. Maxwell (eds.): Scientific explanation, space, and time. Minnesota Studies in the philosophy of science. Volume 3. Minneapolis: University of Minnesota Press. Pp. 28-97.

Field, Hartry H. (2003): "Causation in a physical world”. In: M. Loux \& D. Zimmerman (eds.): The Oxford handbook of metaphysics. Oxford: Oxford University Press. Pp. 435-460.

French, Steven \& Ladyman, James (2003): "Remodelling structural realism: quantum physics and the metaphysics of structure". Synthese 136, pp. 31-56.

Ghirardi, Giancarlo, Rimini, Alberto \& Weber, Tullio (1986): "Unified dynamics for microscopic and macroscopic systems”. Physical Review D34, pp. 470-491.

Gilmore, Cody S. (2002): "Balashov on special relativity and temporal parts”. Philosophical Studies 109, pp. 241-263.

Hales, Steven D. \& Johnson, Timothy A. (2003): "Endurantism, perdurantism, and special relativity". Philosophical Quarterly 53, pp. 524-539.

Harré, Rom \& Madden, E. H. (1975): Causal powers. A theory of natural necessity. Oxford: Blackwell.

Hawley, Katherine (2006): “Science as a guide to metaphysics?” Synthese 149, pp. 451-470.

Hooker, Clifford A. (1981): "Towards a general theory of reduction. Part I: Historical and scientific setting. Part II: Identity in reduction. Part III: Cross-categorial reduction”. Dialogue 20, pp. 38-60, 201-236, 496-529.

Hooker, Clifford A. (2004): “Asymptotics, reduction and emergence”. British Journal for the Philosophy of Science 55, pp. 435-479.

Howard, Don (1989): "Holism, separability, and the metaphysical implications of the Bell experiments". In: J. T. Cushing \& E. McMullin (eds.): Philosophical consequences of quantum theory. Reflections on Bell's theorem. Notre Dame: University of Notre Dame Press. Pp. 224-253.

Huggett, Nick \& Weingard, Robert (1994): "Interpretations of quantum field theory". Philosophy of Science 61, pp. 370-388.

Kuhn, Thomas S. (1962): The structure of scientific revolutions. Chicago: University of Chicago Press. 
Ladyman, James \& Ross, Don with Spurrett, David \& Collier, John (2007): Every thing must go. Metaphysics naturalised. Oxford: Oxford University Press.

Lam, Vincent (2005): “Causation and space-time”. History and Philosophy of the Life Sciences 27, pp. 457-470.

Langton, Rae \& Lewis, David (1998): “Defining “intrinsic'”. Philosophy and Phenomenological Research 58, pp. 333-345. Reprinted in David Lewis (1999): Papers in metaphysics and epistemology. Cambridge: Cambridge University Press. Pp. 116-132.

Laudan, Larry (1981): “A confutation of convergent realism”. Philosophy of Science 48, pp. 19-49. Reprinted in D. Papineau (ed.) (1996): The philosophy of science. Oxford: Oxford University Press. Pp. 107-138.

Laudan, Larry (1984): “Realism without the real”. Philosophy of Science 51, pp. 156-162.

Laudan, Larry (1996): Beyond positivism and relativism. Theory, method, and evidence. Boulder: Westview Press.

Laudan, Larry \& Leplin, Jarrett (1991): “Empirical equivalence and underdetermination”. Journal of Philosophy 88, pp. 449-472.

Lockwood, Michael (1989): Mind, brain and the quantum. The compound 'I'. Oxford: Blackwell.

Lyre, Holger (2004): Lokale Symmetrien und Wirklichkeit. Eine naturphilosophische Studie über Eichtheorien und Strukturenrealismus. Paderborn: Mentis.

Maudlin, Tim (2007): The metaphysics in physics. Oxford: Oxford University Press.

Mumford, Stephen (2006): “The ungrounded argument”. Synthese 149, pp. 471-489.

Norton, John (2007): “Causation as folk science”. In: H. Price \& R. Corry (eds.) (2007): Causation, physics, and the constitution of reality. Russell's republic revisited. Oxford: Oxford University Press. Pp. 11-44.

Papineau, David (1996): "Introduction”. In: D. Papineau (ed.): The philosophy of science. Oxford: Oxford University Press. Pp. 1-20.

Popper, Karl R. (1959): The logic of scientific discovery. London: Hutchinson.

Psillos, Stathis (2006): “What do powers do when they are not manifested?". Philosophy and Phenomenological Research 72, pp. 137-156.

Quine, Willard Van Orman (1953): “Two dogmas of empiricism”. In: W. V. O. Quine: From a logical point of view. Cambridge (Massachusetts): Harvard University Press. Pp. 20-46.

Rueger, Alexander (1998): "Local theories of causation and the a posteriori identification of the causal relation". Erkenntnis 48, pp. 25-38.

Russell, Bertrand (1912): “On the notion of cause”. Proceedings of the Aristotelian Society 13, pp. 1-26.

Salmon, Wesley C. (1998): Causality and explanation. Oxford: Oxford University Press.

Saunders, Simon (2002): "How relativity contradicts presentism”. In: C. Callender (ed.): Time, reality \& experience. Cambridge: Cambridge University Press. Pp. 277-292.

Schaffner, Kenneth F. (1967): “Approaches to reduction”. Philosophy of Science 34, pp. 137-147.

Shoemaker, Sydney (1980): "Causality and properties”. In: P. van Inwagen (ed.): Time and cause. Dordrecht: Reidel. Pp. 109-135. Reprinted in Sydney Shoemaker (1984): Identity, cause, and mind. Philosophical essays. Cambridge: Cambridge University Press. Pp. 206-233.

Sider, Theodore R. (2001): Four-dimensionalism. Oxford: Clarendon Press.

Sparber, Georg (2006): “Powerful causation”. In: M. Esfeld (ed.): John Heil. Symposium on his ontological point of view. Frankfurt (Main): Ontos. Pp. 123-137.

Sparber, Georg (2009): Unorthodox Humeanism. Frankfurt (Main): Ontos.

Suárez, Mauricio (2007): “Quantum propensities”. Studies in History and Philosophy of Modern Physics 38B, pp. 418-438.

Teller, Paul (1986): "Relational holism and quantum mechanics". British Journal for the Philosophy of Science 37, pp. 71-81.

von Neumann, Johann (1932): Mathematische Grundlagen der Quantenmechanik. Berlin: Springer.

von Neumann, Johann (1955): Mathematical foundations of quantum mechanics. Translated by R. T. Beyer. Princeton: Princeton University Press. 